\title{
Article \\ Multiple Critical Points for Symmetric Functionals without upper Growth Condition on the Principal Part
}

\author{
Marco Degiovanni ${ }^{*}+{ }^{+}$and Marco Marzocchi ${ }^{\dagger}$ \\ Dipartimento di Matematica e Fisica, Università Cattolica del Sacro Cuore, Via Trieste 17, 25121 Brescia, Italy; \\ marco.marzocchi@unicatt.it \\ * Correspondence: marco.degiovanni@unicatt.it \\ † These authors contributed equally to this work.
}

check for updates

Citation: Degiovanni, M.; Marzocchi, M. Multiple Critical Points for Symmetric Functionals without upper Growth Condition on the Principal Part. Symmetry 2021, 13, 898. https:// doi.org/10.3390/sym13050898

Academic Editor: Antonio Masiello

Received: 28 April 2021

Accepted: 13 May 2021

Published: 18 May 2021

Publisher's Note: MDPI stays neutral with regard to jurisdictional claims in published maps and institutional affiliations.

Copyright: (c) 2021 by the authors. Licensee MDPI, Basel, Switzerland. This article is an open access article distributed under the terms and conditions of the Creative Commons Attribution (CC BY) license (https:// creativecommons.org/licenses/by/ $4.0 /)$.
Abstract: This paper is concerned with variational methods applied to functionals of the calculus of variations in a multi-dimensional case. We prove the existence of multiple critical points for a symmetric functional whose principal part is not subjected to any upper growth condition. For this purpose, nonsmooth variational methods are applied.

Keywords: calculus of variations; quasilinear elliptic equations; nonsmooth variational methods

MSC: 35J62; 58E05

\section{Introduction}

Let $\Omega$ be a bounded and open subset of $\mathbb{R}^{n}$. We aim to prove the existence of multiple critical points, in a suitable sense, for a homogeneous Dirichlet problem associated with a functional of the form

$$
f(u)=\int_{\Omega} \Psi(x, \nabla u) d x-\int_{\Omega} G(x, u) d x,
$$

under assumptions that do not guarantee any upper growth condition on the principal part $\Psi(x, \cdot)$.

If $\Psi$ and $G$ are smooth and subjected to suitable growth estimates, the functional $f$ is of class $C^{1}$ on some Sobolev space $W_{0}^{1, p}(\Omega)$, and standard variational methods apply (see e.g., $[1,2])$.

The case in which the growth conditions on $G$ are relaxed, meaning that $f$ is only continuous or even lower semicontinuous, has been already considered in [3,4], but standard growth conditions on the principal part $\Psi$ are still imposed.

However, situations in which there is no upper growth condition on the principal part appear, for instance, in continuum mechanics, and a case in which $\Omega$ is one-dimensional has already been treated in [5]. On the other hand, to the best of our knowledge, in the multi-dimensional case, only the existence of minima has been proved thus far.

Let us also point out that the fact that each minimum satisfies the associated EulerLagrange equation can be not at all obvious. See, e.g., the survey paper [6]. This problem has also been addressed in [7], and the assumptions we will impose on $\Psi$ are related to those required in [7].

In order to prove the existence of minima, the case in which the functional $f$ is coercive is usually considered. As a first step in the direction of existence results for critical points, we will also consider a coercive case. When standard growth conditions on $\Psi$ and $G$ are satisfied, the existence of multiple critical points in the coercive case has been obtained, for instance, in [1,8]. We will prove a result in the line of ([1] Theorem 9.10) adapted to our setting. 
More precisely, we assume that

$$
\Psi: \Omega \times \mathbb{R}^{n} \rightarrow \mathbb{R}, \quad G: \Omega \times \mathbb{R} \rightarrow \mathbb{R}
$$

satisfy

$\left(\Psi G_{1}\right)$ for every $\xi \in \mathbb{R}^{n}$, the function $\{x \mapsto \Psi(x, \xi)\}$ is measurable and, for a.e. $x \in \Omega$, the function $\{\xi \mapsto \Psi(x, \xi)\}$ is convex;

for every $s \in \mathbb{R}$, the function $\{x \mapsto G(x, s)\}$ is measurable and, for a.e. $x \in \Omega$, the function $\{s \mapsto G(x, s)\}$ is of class $C^{1}$; we set $g(x, s)=D_{s} G(x, s)$;

$\left(\Psi G_{2}\right) \quad \Psi(x, 0)=G(x, 0)=0$ for a.a. $x \in \Omega$ and, for every $R>0$, there exist $\alpha_{R}^{(0)} \in L_{\text {loc }}^{1}(\Omega)$ and $\alpha_{R}^{(1)} \in L^{1}(\Omega)$ such that

$$
\Psi(x, \xi) \leq \alpha_{R}^{(0)}(x), \quad|g(x, s)| \leq \alpha_{R}^{(1)}(x),
$$

for a.a. $x \in \Omega$ and all $s \in \mathbb{R}, \xi \in \mathbb{R}^{n}$ with $|s| \leq R,|\xi| \leq R$;

$\left(\Psi G_{3}\right)$ for every $M>0$, there exists $\alpha_{M}^{(2)} \in L^{1}(\Omega)$ such that

$$
\Psi(x, \xi) \geq M|\xi|-\alpha_{M}^{(2)}(x), \quad \text { for a.a. } x \in \Omega \text { and all } \xi \in \mathbb{R}^{n} ;
$$

moreover, if $n \geq 2$, there exist $\alpha^{(3)} \in L^{1}(\Omega)$ and $\beta \in \mathbb{R}$ such that

$$
s g(x, s) \leq \alpha^{(3)}(x)+\beta|s|^{\frac{n}{n-1}}, \quad \text { for a.a. } x \in \Omega \text { and all } s \in \mathbb{R} ;
$$

$\left(G_{4}\right) \quad$ there exist $\hat{\alpha} \in L^{1}(\Omega)$ and $\hat{\beta} \in L^{n}(\Omega)$ such that

$$
G(x, s) \leq \hat{\alpha}(x)+\hat{\beta}(x)|s| \quad \text { for a.a. } x \in \Omega \text { and all } s \in \mathbb{R} ;
$$

moreover, if $n \geq 2$, there exists also $\tilde{\beta} \in \mathbb{R}$ such that

$$
G(x, s) \geq-\hat{\alpha}(x)-\tilde{\beta}|s|^{\frac{n}{n-1}} \quad \text { for a.a. } x \in \Omega \text { and all } s \in \mathbb{R} .
$$

Remark 1. According to ([9] Corollary 2.3), it follows that the function $\{\xi \mapsto \Psi(x, \xi)\}$ is continuous for a.a. $x \in \Omega$. Moreover, we have

$$
|g(x, s)| \leq \alpha_{1}^{(1)}(x)+|g(x, s) s| \quad \text { for a.a. } x \in \Omega \text { and all } s \in \mathbb{R},
$$

where $\alpha_{1}^{(1)} \in L^{1}(\Omega)$ is given by assumption $\left(\Psi G_{2}\right)$.

Given $\lambda>0$, we can define a functional

$$
\left.\left.f_{\lambda}: L^{\frac{n}{n-1}}(\Omega) \rightarrow\right]-\infty,+\infty\right]
$$

by

$$
f_{\lambda}(u)= \begin{cases}\int_{\Omega} \Psi(x, \nabla u) d x-\lambda \int_{\Omega} G(x, u) d x & \text { if } u \in W_{0}^{1,1}(\Omega), \\ +\infty & \text { otherwise, }\end{cases}
$$

where we agree that $L^{n /(n-1)}(\Omega)=L^{\infty}(\Omega)$ in the case $n=1$.

Remark 2. By standard results, the functional

$$
u \mapsto \begin{cases}\int_{\Omega} \Psi(x, \nabla u) d x & \text { if } u \in W_{0}^{1,1}(\Omega), \\ +\infty & \text { otherwise, }\end{cases}
$$


is convex and lower semicontinuous on $L^{n /(n-1)}(\Omega)$ (see also the next Corollary cor:lsc), while the functional

$$
u \mapsto \int_{\Omega} G(x, u) d x
$$

is continuous on $L^{n /(n-1)}(\Omega)$. However, it is not locally Lipschitz, unless $n=1$, as we do not have a convenient estimate of $|g(x, s)|$.

Let us point out that we need to consider the functional $f_{\lambda}$ on a Lebesgue space such as $L^{n /(n-1)}(\Omega)$ and not, e.g., on $W_{0}^{1,1}(\Omega)$, because $\Psi(x, \cdot)$ is not assumed to be strictly convex and, consequently, it is impossible to prove a Palais-Smale condition related to a norm which requires the strong convergence of $\nabla u$.

Definition 1. Let $\lambda>0$. A function $u \in W_{0}^{1,1}(\Omega)$ is said to be an energy critical point of the functional $f_{\lambda}$, if

$$
\Psi(x, \nabla u) \in L^{1}(\Omega), \quad G(x, u) \in L^{1}(\Omega), \quad g(x, u) u \in L^{1}(\Omega),
$$

and $u$ is a minimum of the convex functional

$$
\left\{v \mapsto \int_{\Omega} \Psi(x, \nabla v) d x-\lambda \int_{\Omega} g(x, u) v d x\right\}
$$

defined on the linear space

$$
V_{u}:=\left\{v \in W_{0}^{1,1}(\Omega): g(x, u) v \in L^{1}(\Omega)\right\}
$$

Remark 3. According to Remark 1, we have that $g(x, u) u \in L^{1}(\Omega)$ implies $g(x, u) \in L^{1}(\Omega)$. Therefore, we have $u \in V_{u}$ and $W_{0}^{1,1}(\Omega) \cap L^{\infty}(\Omega) \subseteq V_{u}$.

Let us state our main result.

Theorem 1. Assume that $\Psi(x, \cdot)$ and $G(x, \cdot)$ are even for a.a. $x \in \Omega$ and that there exists $r>0$ such that $G(x, s)>0$ for a.a. $x \in \Omega$ and all $s \in \mathbb{R}$ with $0<|s|<r$.

Then, for every $m \geq 1$, there exists $\Lambda_{m}>0$ such that, for every $\lambda \geq \Lambda_{m}$, the functional $f_{\lambda}$ possesses at least $m$ distinct pairs $\left(u_{1},-u_{1}\right), \ldots,\left(u_{m},-u_{m}\right)$ of energy critical points in $W_{0}^{1,1}(\Omega)$ with $f_{\lambda}\left(u_{j}\right)<0$ for all $j=1, \ldots, m$.

Since we are mainly interested in the principal part of the functional, in the next examples, we propose the same lower-order term, even if other choices are possible.

Concerning the principal part, since there is no upper bound on $\Psi(x, \cdot)$, one can consider, in particular, cases with nonstandard growth conditions.

Example 1. The assumptions of Theorem 1 are satisfied by a functional of the form

$$
u \mapsto \int_{\Omega} a_{0}(x) \vartheta(\nabla u) d x-\lambda \int_{\Omega} a_{1}(x) \arctan \left(u^{2}\right) d x
$$

where $a_{0} \in L_{\text {loc }}^{1}(\Omega), a_{1} \in L^{1}(\Omega)$ are such that

$$
a_{0}(x) \geq 1, \quad a_{1}(x)>0, \quad \text { for a.a. } x \in \Omega
$$

and $\vartheta: \mathbb{R}^{n} \rightarrow \mathbb{R}$ is convex, even and satisfies

$$
\vartheta(0)=0, \quad \lim _{|\xi| \rightarrow+\infty} \frac{\vartheta(\xi)}{|\xi|}=+\infty .
$$


Let us point out that, in the case $n=2$, a possible choice is

$$
\vartheta\left(\xi_{1}, \xi_{2}\right)=\left|\xi_{1}\right| \log \left(e+\left|\xi_{1}\right|\right)+\exp \left(\xi_{2}^{2}\right)-1,
$$

with a very different behavior in the the two variables $\xi_{1}$ and $\xi_{2}$.

Let us also point out that, if $n \geq 2$, the functional

$$
u \mapsto \int_{\Omega} a_{1}(x) \arctan \left(u^{2}\right) d x
$$

is continuous on $L^{n /(n-1)}(\Omega)$, but not locally Lipschitz, unless further summability conditions on $a_{1}$ are imposed.

Example 2. The assumptions of Theorem 1 are satisfied by the functionals

$$
\begin{aligned}
u & \mapsto \int_{\Omega} c(x)|\nabla u|^{p} d x-\lambda \int_{\Omega} a_{1}(x) \arctan \left(u^{2}\right) d x, \\
u & \mapsto \int_{\Omega}|\nabla u|^{p(x)} d x-\lambda \int_{\Omega} a_{1}(x) \arctan \left(u^{2}\right) d x, \\
u & \mapsto \int_{\Omega}\left[|\nabla u|^{q}+d(x)|\nabla u|^{r}\right] d x-\lambda \int_{\Omega} a_{1}(x) \arctan \left(u^{2}\right) d x,
\end{aligned}
$$

with $a_{1}$ as in the previous example and

$$
\begin{gathered}
p>1, c \in L_{l o c}^{1}(\Omega), c>0 \text { a.e. in } \Omega \text { and } c^{-1 /(p-1)} \in L^{1}(\Omega), \\
\quad p(x) \in L_{l o c}^{\infty}(\Omega) \text { with } \underset{x \in \Omega}{\operatorname{ess} \inf } p(x)>1 \\
q>1, r \geq 1 \text { and } d \in L_{l o c}^{1}(\Omega) \text { with } d \geq 0 \text { a.e. in } \Omega .
\end{gathered}
$$

Principal parts of this form appear, for instance, in the study of strongly nonhomogeneous materials and non-Newtonian fluids (see, e.g., [10-12] and references therein).

Concerning the first case, let us recall that, by Young's inequality, one has

$$
\begin{aligned}
M|\xi| & =\left[p^{1 / p} c(x)^{1 / p}|\xi|\right]\left[M p^{-1 / p} c(x)^{-1 / p}\right] \\
& \leq c(x)|\xi|^{p}+\frac{p-1}{p^{p /(p-1)}} M^{p /(p-1)} c(x)^{-1 /(p-1)},
\end{aligned}
$$

whence

$$
c(x)|\xi|^{p} \geq M|\xi|-\frac{p-1}{p^{p /(p-1)}} M^{p /(p-1)} c(x)^{-1 /(p-1)}
$$

and assumption $\left(\Psi G_{3}\right)$ follows.

Under a smoothness assumption on $\Psi(x, \cdot)$, we have that each energy critical point is also a weak solution of the associated Euler-Lagrange equation.

Proposition 1. Let $\lambda>0$ and let $u \in W_{0}^{1,1}(\Omega)$ be an energy critical point of $f_{\lambda}$. Assume also that, for a.e. $x \in \Omega$, the function $\Psi(x, \cdot)$ is of class $C^{1}$.

Then

$$
\nabla_{\xi} \Psi(x, \nabla u) \in L_{l o c}^{1}\left(\Omega ; \mathbb{R}^{n}\right), \quad \nabla_{\xi} \Psi(x, \nabla u) \cdot \nabla u \in L^{1}(\Omega),
$$

and we have

$$
\begin{aligned}
& \int_{\Omega} \nabla_{\xi} \Psi(x, \nabla u) \cdot \nabla v d x=\lambda \int_{\Omega} g(x, u) v d x \quad \text { for all } v \in C_{c}^{1}(\Omega), \\
& \int_{\Omega} \nabla_{\xi} \Psi(x, \nabla u) \cdot \nabla u d x \leq \lambda \int_{\Omega} g(x, u) u d x .
\end{aligned}
$$


Moreover, we also have

$$
\begin{aligned}
& \nabla_{\xi} \Psi(x, \nabla u) \cdot \nabla v \in L^{1}(\Omega), \\
& \int_{\Omega} \nabla_{\xi} \Psi(x, \nabla u) \cdot(\nabla v-\nabla u) d x \geq \lambda \int_{\Omega} g(x, u)(v-u) d x, \\
& \quad \text { for all } v \in W_{0}^{1,1}(\Omega) \text { with } \Psi(x, \nabla v) \in L^{1}(\Omega) \text { and }[g(x, u) v]^{-} \in L^{1}(\Omega) .
\end{aligned}
$$

In Section 2, we recall the tools of nonsmooth critical point theory we need. In Section 3, we adapt some basic results from [13] to our setting. The main technical results are contained in Section 4, where we show how the nonsmooth critical point theory can be applied to a functional such as $f_{\lambda}$. Since we believe that the approach can be useful also when the functional is not coercive, in Section 4 , assumption $\left(G_{4}\right)$ is replaced by more general hypotheses. Finally, in Section 5, we prove the results stated in the Introduction.

In the following, we will denote by $\|\cdot\|_{p}$ the usual norm in $L^{p}$. For every $s \in \mathbb{R}$, we also set

$$
s^{ \pm}=\max \{ \pm s, 0\}, \quad T_{k}(s)=\min \{\max \{s,-k\}, k\} .
$$

\section{Nonsmooth Critical Point Theory}

In this section, we recall some useful tools. We refer the reader to [5,14-18] for proofs and more details.

Let $X$ be a metric space endowed with the distance $d$. We denote by $\mathrm{B}_{\delta}(u)$ the open ball of center $u$ and radius $\delta$. We will also consider the set $X \times \mathbb{R}$ endowed with the distance

$$
d((u, \tau),(v, \eta))=\left(d(u, v)^{2}+(\tau-\eta)^{2}\right)^{1 / 2}
$$

Let $f: X \rightarrow[-\infty,+\infty]$ be a function and let

$$
\operatorname{epi}(f)=\{(u, \tau) \in X \times \mathbb{R}: f(u) \leq \tau\} .
$$

When $f$ is real-valued and continuous, the next notion has been independently introduced in [15,16] and in [18], while a variant has been developed in [17]. By means of a suitable device, also the general case was considered in $[15,16]$. Here, we follow the equivalent approach of [14].

Definition 2. For every $u \in X$ with $f(u) \in \mathbb{R}$, we denote by $|d f|(u)$ the supremum of the $\sigma^{\prime}$ s in $[0,+\infty[$ such that there exist $\delta>0$ and a continuous map

$$
\mathcal{H}:\left(\mathrm{B}_{\delta}(u, f(u)) \cap \operatorname{epi}(f)\right) \times[0, \delta] \rightarrow X
$$

satisfying

$$
d(\mathcal{H}((w, \tau), t), w) \leq t, \quad f(\mathcal{H}((w, \tau), t)) \leq \tau-\sigma t
$$

whenever $(w, \tau) \in \mathrm{B}_{\delta}(u, f(u)) \cap \operatorname{epi}(f)$ and $t \in[0, \delta]$.

The extended real number $|d f|(u)$ is called the weak slope of $f$ at $u$.

When $f$ is real-valued and continuous, the next result provides a simple estimate.

Proposition 2. Let $f: X \rightarrow \mathbb{R}$ be a continuous function. Assume there exist $\sigma \geq 0, \varrho>0, \delta>0$ and a continuous map

$$
\mathcal{H}: \mathrm{B}_{\delta}(u) \times[0, \delta] \rightarrow X
$$

satisfying

$$
d(\mathcal{H}(w, t), w) \leq \varrho t, \quad f(\mathcal{H}(w, t)) \leq f(w)-\sigma t,
$$

whenever $w \in \mathrm{B}_{\delta}(u)$ and $t \in[0, \delta]$.

Then, we have $|d f|(u) \geq \sigma / \varrho$. 
Proof. It is a simple consequence of ([14] Proposition 2.2).

Remark 4. Let $X$ be an open subset of a normed space and let $f: X \rightarrow \mathbb{R}$ be of class $C^{1}$. Then, we have $|d f|(u)=\left\|f^{\prime}(u)\right\|$ for all $u \in X$.

Definition 3. We say that $u \in X$ is a (lower) critical point of $f$ if $f(u) \in \mathbb{R}$ and $|d f|(u)=0$. We say that $c \in \mathbb{R}$ is $a$ (lower) critical value of $f$ if there exists $u \in X$ such that $f(u)=c$ and $|d f|(u)=0$.

Definition 4. Let $c \in \mathbb{R}$. A sequence $\left(u_{k}\right)$ in $X$ is said to be a Palais-Smale sequence at level $c$ $\left((P S)_{c}\right.$-sequence, for short) for $f$, if

$$
\lim _{k} f\left(u_{k}\right)=c, \quad \lim _{k}|d f|\left(u_{k}\right)=0 .
$$

We say that $f$ satisfies the Palais-Smale condition at level $c\left((P S)_{c}\right.$, for short), if every $(P S)_{c}$-sequence for $f$ admits a convergent subsequence in $X$.

Now, let us see, following $[15,16]$, how the case of a general $f$ can be reduced, to some extent, to the continuous case, taking advantage of the function $\mathcal{G}_{f}$ introduced in [19].

Define a function

$$
\mathcal{G}_{f}: \operatorname{epi}(f) \rightarrow \mathbb{R}
$$

by $\mathcal{G}_{f}(u, \tau)=\tau$. It is readily seen that $\mathcal{G}_{f}$ is Lipschitz continuous of constant 1 , whence $\left|d \mathcal{G}_{f}\right|(u, \tau) \leq 1$ for all $(u, \tau) \in \operatorname{epi}(f)$.

Proposition 3. For every $u \in X$ with $f(u) \in \mathbb{R}$, we have

$$
|d f|(u)= \begin{cases}\frac{\left|d \mathcal{G}_{f}\right|(u, f(u))}{\sqrt{1-\left|d \mathcal{G}_{f}\right|(u, f(u))^{2}}} & \text { if }\left|d \mathcal{G}_{f}\right|(u, f(u))<1, \\ +\infty & \text { if }\left|d \mathcal{G}_{f}\right|(u, f(u))=1 .\end{cases}
$$

Proof. See ([14] Proposition 2.3).

We aim to reduce the study of a general $f$ to that of the continuous function $\mathcal{G}_{f}$. In view of the natural correspondence $u \leftrightarrow(u, f(u))$, a key point is to have a control on pairs $(u, \tau) \in \operatorname{epi}(f)$ with $f(u)<\tau$.

Definition 5. Let $c \in \mathbb{R}$. We say that $f$ satisfies condition $(\text { epi })_{c}$, if there exists $\varepsilon>0$ such that

$$
\inf \left\{\left|d \mathcal{G}_{f}\right|(u, \tau):(u, \tau) \in \operatorname{epi}(f), f(u)<\tau,|\tau-c|<\varepsilon\right\}>0 .
$$

Remark 5. If $f: X \rightarrow \mathbb{R}$ is continuous, then $\left|d \mathcal{G}_{f}\right|(u, \tau)=1$ whenever $f(u)<\tau$.

Proof. See ([16] Proposition 2.3).

Several results of critical point theory can be extended to the nonsmooth case, by means of the previous concepts. In view of our purposes, let us mention an extension of D.C. Clark's theorem (see $[1,8]$ when $f$ is smooth).

Theorem 2. Let $X$ be a Banach space, let $f: X \rightarrow]-\infty,+\infty]$ be a lower semicontinuous function and let $m \geq 1$. Assume that:

(a) The function $f$ is even and bounded from below; 
(b) There exists an odd and continuous map $\psi$ from the $(m-1)$-dimensional sphere $S^{m-1}$ to $X$ such that

$$
\sup _{\psi\left(S^{m-1}\right)} f<f(0)
$$

(c) For every $c<f(0)$, the function $f$ satisfies $(P S)_{c}$ and $(\text { epi })_{c}$.

Then, there exist at least $m$ distinct pairs $\left(u_{1},-u_{1}\right), \ldots,\left(u_{m},-u_{m}\right)$ of critical points of $f$ with $f\left(u_{j}\right)<f(0)$ for all $j=1, \ldots, m$.

Proof. See ([5] Theorem 2.5).

When dealing with the weak slope $|d f|(u)$, an auxiliary concept is sometimes useful. From now on in this section, we assume that $X$ is a normed space over $\mathbb{R}$ and $f: X \rightarrow$ $[-\infty,+\infty]$ is a function.

The next notion has been introduced in [14].

Definition 6. For every $u \in X$ with $f(u) \in \mathbb{R}, v \in X$ and $\varepsilon>0$, let $f_{\varepsilon}^{0}(u ; v)$ be the infimum of $r^{\prime}$ s in $\mathbb{R}$ such that there exist $\delta>0$ and a continuous map

$$
\left.\left.\mathcal{V}:\left(\mathrm{B}_{\delta}(u, f(u)) \cap \operatorname{epi}(f)\right) \times\right] 0, \delta\right] \rightarrow \mathrm{B}_{\varepsilon}(v)
$$

satisfying

$$
f(w+t \mathcal{V}((w, \tau), t)) \leq \tau+r t
$$

whenever $(w, \tau) \in B_{\delta}(u, f(u)) \cap \operatorname{epi}(f)$ and $\left.\left.t \in\right] 0, \delta\right]$.

Then, let

$$
f^{0}(u ; v)=\sup _{\varepsilon>0} f_{\varepsilon}^{0}(u ; v)=\lim _{\varepsilon \rightarrow 0} f_{\varepsilon}^{0}(u ; v) .
$$

Let us recall that the function $f^{0}(u ; \cdot): X \rightarrow[-\infty,+\infty]$ is convex, lower semicontinuous and positively homogeneous of degree 1.

Definition 7. For every $u \in X$ with $f(u) \in \mathbb{R}$, we set

$$
\partial f(u)=\left\{\mu \in X^{\prime}:\langle\mu, v\rangle \leq f^{0}(u ; v) \text { for all } v \in X\right\} .
$$

Remark 6. If $f$ is convex, then $\partial f$ agrees with the subdifferential of convex analysis. If $f$ is locally Lipschitz, then $f^{0}$ and $\partial f$ agree with Clarke's notions [20], while, in general, $\partial_{C} f(u) \subseteq \partial f(u)$, where $\partial_{C} f(u)$ denotes Clarke's subdifferential.

The subdifferential we have recalled is suitably related to the weak slope because of the next result.

Theorem 3. For every $u \in X$ with $f(u) \in \mathbb{R}$, the following facts hold:

(a) $|d f|(u)<+\infty \Longleftrightarrow \partial f(u) \neq \varnothing \Longleftrightarrow f^{0}(u ; 0)>-\infty$;

(b) $\quad|d f|(u)<+\infty \Longrightarrow|d f|(u) \geq \min \{\|\mu\|: \mu \in \partial f(u)\}$.

Proof. See ([14] Theorem 4.13).

\section{Compactness and Lower Semicontinuity}

Throughout this section, we consider the more general situation in which

$$
\left.\left.f: L^{\frac{n}{n-1}}(\Omega) \rightarrow\right]-\infty,+\infty\right]
$$


has the form

$$
f(u)= \begin{cases}\int_{\Omega} L(x, u, \nabla u) d x & \text { if } u \in W_{0}^{1,1,}(\Omega), \\ +\infty & \text { otherwise. }\end{cases}
$$

We assume that

$$
L: \Omega \times \mathbb{R} \times \mathbb{R}^{n} \rightarrow \mathbb{R}
$$

satisfies

$\left(L_{1}\right) \quad$ for every $(s, \xi) \in \mathbb{R} \times \mathbb{R}^{n}$, the function $\{x \mapsto L(x, s, \xi)\}$ is measurable and, for a.e. $x \in \Omega$, the function $\{(s, \xi) \mapsto L(x, s, \xi)\}$ is continuous; moreover, for a.e. $x \in \Omega$ and every $s \in \mathbb{R}$, the function $\{\xi \mapsto L(x, s, \xi)\}$ is convex;

- if $n \geq 2$, there exist $\beta \in \mathbb{R}$ and, for every $M>0, \alpha_{M} \in L^{1}(\Omega)$ such that

$$
L(x, s, \xi) \geq M|\xi|-\alpha_{M}(x)-\beta|s|^{\frac{n}{n-1}}
$$

for a.a. $x \in \Omega$ and all $(s, \xi) \in \mathbb{R} \times \mathbb{R}^{n}$;

- if $n=1$, for every $M, R>0$ there exists $\alpha_{M, R} \in L^{1}(\Omega)$ such that

$$
L(x, s, \xi) \geq M|\xi|-\alpha_{M, R}(x)
$$

for a.a. $x \in \Omega$ and all $(s, \xi) \in \mathbb{R} \times \mathbb{R}^{n}$ with $|s| \leq R$. defined.

Since $W_{0}^{1,1}(\Omega) \subseteq L^{n /(n-1)}(\Omega)$, under these assumptions, the functional $f$ is well

Theorem 4. Let $\left(u_{k}\right)$ be a sequence in $W_{0}^{1,1}(\Omega)$ such that

$$
\sup _{k}\left\|u_{k}\right\|_{\frac{n}{n-1}}<+\infty, \quad \sup _{k} f\left(u_{k}\right)<+\infty .
$$

Then, there exist $u \in W_{0}^{1,1}(\Omega)$ and a subsequence $\left(u_{k_{j}}\right)$ weakly converging to $u$ in $W_{0}^{1,1}(\Omega)$ with

$$
\lim _{j}\left\|u_{k_{j}}-u\right\|_{\frac{n}{n-1}}=0, \quad \liminf _{j} f\left(u_{k_{j}}\right) \geq f(u) .
$$

Proof. Suppose, first, $n \geq 2$. From $\left(L_{2}\right)$, we infer that there exist $\alpha_{1} \in L^{1}(\Omega)$ and $\beta \in \mathbb{R}$ such that

$$
L(x, s, \xi) \geq|\xi|-\alpha_{1}(x)-\beta|s|^{\frac{n}{n-1}} .
$$

By replacing $L(x, s, \xi)$ with

$$
\widetilde{L}(x, s, \xi)=L(x, s, \xi)+\alpha_{1}(x)+\beta|s|^{\frac{n}{n-1}},
$$

we may assume, without loss of generality, that $\left(L_{2}\right)$ is satisfied with $\beta=0$ and that $L(x, s, \xi) \geq|\xi|$.

If $n=1$, let

$$
R=\sup _{k}\left\|u_{k}\right\|_{\infty} .
$$

From $\left(L_{2}\right)$, we infer that there exists $\alpha_{1, R} \in L^{1}(\Omega)$ such that

$$
L(x, s, \xi) \geq|\xi|-\alpha_{1, R}(x) \quad \text { whenever }|s| \leq R .
$$

By replacing $L(x, s, \xi)$ with

$$
\widetilde{L}(x, s, \xi)=L(x, s, \xi)+\alpha_{1, R}(x),
$$

we may assume, without loss of generality, that $L(x, s, \xi) \geq|\xi|$ whenever $|s| \leq R$. 
In both cases, $n \geq 2$ and $n=1$, we infer that $\left(u_{k}\right)$ is bounded in $W_{0}^{1,1}(\Omega)$, hence convergent, up to a subsequence we still denote by $\left(u_{k}\right)$, to some $u$ in $L^{1}(\Omega)$, and that $L\left(x, u_{k}, \nabla u_{k}\right) \geq 0$ a.e. in $\Omega$.

Let $F>0$ be such that

$$
F \geq \sup _{k} f\left(u_{k}\right) .
$$

Again, by $\left(L_{2}\right)$, for every $\varepsilon>0$, there exists $\alpha \in L^{1}(\Omega)$ such that

$$
L\left(x, u_{k}, \nabla u_{k}\right) \geq \frac{5 F}{\varepsilon}\left|\nabla u_{k}\right|-\alpha(x) \quad \text { for all } k \in \mathbb{N} .
$$

Let

$$
\alpha=\tilde{\alpha}+\hat{\alpha},
$$

with $\tilde{\alpha} \in L^{\infty}(\Omega)$ and $\hat{\alpha} \in L^{1}(\Omega)$ satisfying $\|\hat{\alpha}\|_{1} \leq F$, and let $c>0$ be such that $F c \geq \varepsilon\|\tilde{\alpha}\|_{\infty}$. Finally, let $\delta=\varepsilon /(2 c)$.

Then, we have

$$
L\left(x, u_{k}, \nabla u_{k}\right) \geq \frac{4 F}{\varepsilon}\left|\nabla u_{k}\right|-\hat{\alpha}(x) \quad \text { where }\left|\nabla u_{k}\right| \geq c .
$$

It follows that

$$
\begin{aligned}
F & \geq \int_{\Omega} L\left(x, u_{k}, \nabla u_{k}\right) d x \\
& \geq \int_{\left\{\left|\nabla u_{k}\right| \geq c\right\}} L\left(x, u_{k}, \nabla u_{k}\right) d x \\
& \geq \frac{4 F}{\varepsilon} \int_{\left\{\left|\nabla u_{k}\right| \geq c\right\}}\left|\nabla u_{k}\right| d x-\int_{\Omega}|\hat{\alpha}| d x \\
& \geq \frac{4 F}{\varepsilon} \int_{\left\{\left|\nabla u_{k}\right| \geq c\right\}}\left|\nabla u_{k}\right| d x-F,
\end{aligned}
$$

whence

$$
\int_{\left\{\left|\nabla u_{k}\right| \geq c\right\}}\left|\nabla u_{k}\right| d x \leq \frac{\varepsilon}{2} \quad \text { for all } k \in \mathbb{N} .
$$

If $E$ is a measurable subset of $\Omega$ such that $\mathcal{L}^{n}(E)<\delta$, then for every $k \in \mathbb{N}$, we have

$$
\begin{aligned}
\int_{E}\left|\nabla u_{k}\right| d x & \leq \int_{E \cap\left\{\left|\nabla u_{k}\right|<c\right\}}\left|\nabla u_{k}\right| d x+\int_{\left\{\left|\nabla u_{k}\right| \geq c\right\}}\left|\nabla u_{k}\right| d x \\
& \leq c \mathcal{L}^{n}(E)+\frac{\varepsilon}{2}<\varepsilon .
\end{aligned}
$$

According to ([13] Theorem 1.2.8), we have that $u \in W_{0}^{1,1}(\Omega)$ and that $\left(u_{k}\right)$ is weakly convergent to $u$ in $W_{0}^{1,1}(\Omega)$.

From ([13] Theorem 2.3.1), we infer that

$$
\liminf _{k} \int_{\Omega} L\left(x, u_{k}, \nabla u_{k}\right) d x \geq \int_{\Omega} L(x, u, \nabla u) d x,
$$

whence

$$
\liminf _{k} f\left(u_{k}\right) \geq f(u) .
$$

Moreover, by the Dunford-Pettis property (see, e.g., [21]), it follows that

$$
\lim _{k}\left\|u_{k}-u\right\|_{\frac{n}{n-1}}=0
$$

in the case $n \geq 2$. 
In the case $n=1$, we extend $u_{k}, u$ with value 0 outside $\Omega$, so that $u_{k}, u \in W^{1,1}(\mathbb{R})$, and fix $\tau \in \mathbb{R} \backslash \Omega$. If $\left(t_{k}\right)$ is convergent to $t$ in $\bar{\Omega}$, then

$$
\begin{aligned}
\left|u_{k}\left(t_{k}\right)-u\left(t_{k}\right)\right| & =\left|\int_{\tau}^{t}\left(u_{k}^{\prime}-u^{\prime}\right) d x+\int_{t}^{t_{k}}\left(u_{k}^{\prime}-u^{\prime}\right) d x\right| \\
& \leq\left|\int_{\tau}^{t}\left(u_{k}^{\prime}-u^{\prime}\right) d x\right|+\left|\int_{t}^{t_{k}}\left(\left|u_{k}^{\prime}\right|+\left|u^{\prime}\right|\right) d x\right| .
\end{aligned}
$$

According to the previous step, it follows that

$$
\lim _{k}\left|u_{k}\left(t_{k}\right)-u\left(t_{k}\right)\right|=0 .
$$

Therefore, $\left(u_{k}\right)$ is uniformly convergent to $u$ on $\bar{\Omega}$, whence

$$
\lim _{k}\left\|u_{k}-u\right\|_{\infty}=0
$$

Let us point out two obvious consequences.

Corollary 1. The functional $f$ is lower semicontinuous.

Corollary 2. Let $c \in \mathbb{R}$ and let $\left(u_{k}\right)$ be a $(P S)_{c}$-sequence for $f$ such that

$$
\sup _{k}\left\|u_{k}\right\|_{\frac{n}{n-1}}<+\infty .
$$

Then, there exist $u \in W_{0}^{1,1}(\Omega)$ with $f(u) \leq c$ and a subsequence $\left(u_{k_{j}}\right)$ such that

$$
\lim _{j}\left\|u_{k_{j}}-u\right\|_{\frac{n}{n-1}}=0 .
$$

\section{The Variational Approach}

Throughout this section, we consider two functions, $\Psi$ and $G$, satisfying $\left(\Psi G_{1}\right),\left(\Psi G_{2}\right)$ and $\left(\Psi G_{3}\right)$. Moreover, we assume that:

$\left(G_{5}\right) \quad$ If $n \geq 2$, there exist $\hat{\alpha} \in L^{1}(\Omega)$ and $\hat{\beta} \in \mathbb{R}$ such that

$$
G(x, s) \leq \hat{\alpha}(x)+\hat{\beta}|s|^{\frac{n}{n-1}} \quad \text { for a.a. } x \in \Omega \text { and all } s \in \mathbb{R} .
$$

On the other hand, if $n=1$, it follows from $\left(\Psi G_{2}\right)$ that, for every $R>0$, there exists $\hat{\alpha}_{R} \in L^{1}(\Omega)$ such that

$$
|G(x, s)| \leq \hat{\alpha}_{R}(x) \quad \text { for a.a. } x \in \Omega \text { and all } s \in \mathbb{R} \text { with }|s| \leq R .
$$

In any case, the function $L(x, s, \xi)=\Psi(x, \xi)-G(x, s)$ satisfies $\left(L_{1}\right),\left(L_{2}\right)$ and, according to Corollary 1 , we can define a lower semicontinuous functional

$$
\left.\left.f: L^{\frac{n}{n-1}}(\Omega) \rightarrow\right]-\infty,+\infty\right]
$$

as in (1), namely,

$$
f(u)= \begin{cases}\int_{\Omega} \Psi(x, \nabla u) d x-\int_{\Omega} G(x, u) d x & \text { if } u \in W_{0}^{1,1}(\Omega), \\ +\infty & \text { otherwise. }\end{cases}
$$


According to the Introduction, for every $u \in W_{0}^{1,1}(\Omega)$, we set

$$
V_{u}=\left\{v \in W_{0}^{1,1}(\Omega): g(x, u) v \in L^{1}(\Omega)\right\} .
$$

Lemma 1. If $n \geq 2$, for every $R>0$, there exist $\alpha_{R} \in L^{1}(\Omega)$ and $\beta_{R} \in \mathbb{R}$ such that

$$
\begin{gathered}
g(x, s)(\sigma-s) \geq-\alpha_{R}(x)-\beta_{R}|s|^{\frac{n}{n-1}}, \\
G(x, s+t(\sigma-s)) \geq G(x, s)-t\left(\alpha_{R}(x)+\beta_{R}|s|^{\frac{n}{n-1}}\right),
\end{gathered}
$$

for a.a $x \in \Omega$ and all $0 \leq t \leq 1 / 2$ and $s, \sigma \in \mathbb{R}$ with $|\sigma| \leq R$.

Proof. Let $R>0$. Since

$$
\frac{1}{2} \leq \frac{s-\sigma}{s} \leq \frac{3}{2} \quad \text { whenever }|\sigma| \leq R \text { and }|s| \geq 2 R,
$$

from $\left(\Psi G_{3}\right)$, we infer that

$$
g(x, s)(\sigma-s) \geq-\frac{3}{2} \alpha^{(3)}(x)-\frac{3}{2} \beta|s|^{\frac{n}{n-1}} \quad \text { whenever }|\sigma| \leq R \text { and }|s| \geq 2 R .
$$

Combining this fact with $\left(\Psi G_{2}\right)$, we deduce that there exist $\alpha_{R} \in L^{1}(\Omega)$ and $\beta_{R} \in \mathbb{R}$ such that

$$
g(x, s)(\sigma-s) \geq-\alpha_{R}(x)-\beta_{R}|s|^{\frac{n}{n-1}} \quad \text { for a.a } x \in \Omega \text { and all } s, \sigma \in \mathbb{R} \text { with }|\sigma| \leq R .
$$

Since

$$
\begin{aligned}
G(x, s+t(\sigma-s))-G(x, s) & =\int_{0}^{t} g(x, s+\tau(\sigma-s))(\sigma-s) d \tau \\
& =\int_{0}^{t} \frac{g(x, s+\tau(\sigma-s))[\sigma-s-\tau(\sigma-s)]}{1-\tau} d \tau,
\end{aligned}
$$

there exist also $\tilde{\alpha}_{R} \in L^{1}(\Omega)$ and $\tilde{\beta}_{R} \in \mathbb{R}$ such that

$$
\begin{aligned}
& G(x, s+t(\sigma-s)) \geq G(x, s)-t\left(\tilde{\alpha}_{R}(x)+\tilde{\beta}_{R}|s|^{\frac{n}{n-1}}\right) \\
& \qquad \text { for a.a } x \in \Omega \text { and all } 0 \leq t \leq 1 / 2 \text { and } s, \sigma \in \mathbb{R} \text { with }|\sigma| \leq R
\end{aligned}
$$

and the assertion follows.

Lemma 2. Let $u \in L^{n /(n-1)}(\Omega)$ and let $v \in L^{\infty}(\Omega)$. Then, we have $[g(x, u)(u-v)]^{+} \in L^{1}(\Omega)$ and, for every

$$
r>\int_{\Omega} g(x, u)(u-v) d x
$$

there exists $\delta>0$ such that

$$
\int_{\Omega} G(x, z+t(v-z)) d x \geq \int_{\Omega} G(x, z) d x-r t,
$$

whenever $z \in L^{n /(n-1)}(\Omega),\|z-u\|_{n /(n-1)} \leq \delta$ and $0 \leq t \leq \delta$. 
Proof. Assume, first, that $n \geq 2$. From Lemma 1, we infer that $[g(x, u)(u-v)]^{+} \in L^{1}(\Omega)$. Assume now, for a contradiction, that there exist $t_{k} \rightarrow 0^{+}$and $z_{k} \rightarrow u$ in $L^{n /(n-1)}(\Omega)$ satisfying

$$
\int_{\Omega} G\left(x, z_{k}+t_{k}\left(v-z_{k}\right)\right) d x<\int_{\Omega} G\left(x, z_{k}\right) d x-r t_{k},
$$

whence $G\left(x, z_{k}\right) \in L^{1}(\Omega), t_{k}>0$ and

$$
\int_{\Omega} \frac{G\left(x, z_{k}+t_{k}\left(v-z_{k}\right)\right)-G\left(x, z_{k}\right)}{t_{k}} d x<-r .
$$

Then, from Lemma 1 and the (generalized) Fatou lemma, we infer that

$$
-r \geq \liminf _{k} \int_{\Omega} \frac{G\left(x, z_{k}+t_{k}\left(v-z_{k}\right)\right)-G\left(x, z_{k}\right)}{t_{k}} d x \geq \int_{\Omega} g(x, u)(v-u) d x>-r
$$

and a contradiction follows.

In the case $n=1$, the proof is similar, taking into account assumption $\left(\Psi G_{2}\right)$.

Theorem 5. For every $u \in W_{0}^{1,1}(\Omega)$ with $f(u)<+\infty$, the following facts hold:

(a) We have $\Psi(x, \nabla u) \in L^{1}(\Omega), G(x, u) \in L^{1}(\Omega)$ and

$$
\begin{aligned}
& \int_{\Omega} \Psi(x, \nabla v) d x-\int_{\Omega} \Psi(x, \nabla u) d x-\int_{\Omega} g(x, u)(v-u) d x \geq f^{0}(u ; v-u) \\
& \quad \text { for all } v \in W_{0}^{1,1}(\Omega) \text { with } \Psi(x, \nabla v) \in L^{1}(\Omega) \text { and }[g(x, u)(v-u)]^{-} \in L^{1}(\Omega) ;
\end{aligned}
$$

(b) If $\mu \in \partial f(u)$, then we have that $g(x, u) u \in L^{1}(\Omega), g(x, u) \in L^{1}(\Omega)$ and $u$ is a minimum of the convex functional

$$
\left\{v \mapsto \int_{\Omega} \Psi(x, \nabla v) d x-\int_{\Omega} g(x, u) v d x-\langle\mu, v\rangle\right\}
$$

defined on the convex set

$$
\left\{v \in W_{0}^{1,1}(\Omega): \Psi(x, \nabla v) \in L^{1}(\Omega),[g(x, u) v]^{-} \in L^{1}(\Omega)\right\}
$$

(c) If $\partial f(u)=\varnothing$, then we have $f^{0}(u ; v)=-\infty$ for all $v \in W_{0}^{1,1}(\Omega)$.

Proof. Let $u \in W_{0}^{1,1}(\Omega)$ with $f(u)<+\infty$. From assumptions $\left(\Psi G_{3}\right)$ and $\left(G_{5}\right)$, it readily follows that $\Psi(x, \nabla u) \in L^{1}(\Omega)$ and $G(x, u) \in L^{1}(\Omega)$.

Now, we claim that

$$
\begin{aligned}
& {[g(x, u)(v-u)]^{-} \in L^{1}(\Omega),} \\
& \int_{\Omega} \Psi(x, \nabla v) d x-\int_{\Omega} \Psi(x, \nabla u) d x-\int_{\Omega} g(x, u)(v-u) d x \geq f^{0}(u ; v-u), \\
& \quad \text { for all } v \in W_{0}^{1,1}(\Omega) \cap L^{\infty}(\Omega) \text { with } \Psi(x, \nabla v) \in L^{1}(\Omega) .
\end{aligned}
$$

Actually, Lemma 2 yields $[g(x, u)(v-u)]^{-} \in L^{1}(\Omega)$. Given

$$
r_{1}>\int_{\Omega} \Psi(x, \nabla v) d x-\int_{\Omega} \Psi(x, \nabla u) d x, \quad r_{2}>-\int_{\Omega} g(x, u)(v-u) d x,
$$

we infer from Lemma 2 that there exists $\delta>0$ such that

$$
\int_{\Omega} G(x, z+t(v-z)) d x \geq \int_{\Omega} G(x, z) d x-r_{2} t,
$$


whenever $z \in W_{0}^{1,1}(\Omega),\|z-u\|_{n /(n-1)} \leq \delta$ and $0 \leq t \leq \delta$.

On the other hand, also $\widetilde{L}(x, s, \xi)=\Psi(x, \xi)$ satisfies $\left(L_{1}\right)$ and $\left(L_{2}\right)$, meaning we may assume that

$$
r_{1}>\int_{\Omega} \Psi(x, \nabla v) d x-\int_{\Omega} \Psi(x, \nabla z) d x,
$$

whenever $z \in W_{0}^{1,1}(\Omega)$ and $\|z-u\|_{n /(n-1)} \leq \delta$ by Corollary 1 .

Taking into account the convexity of $\Psi(x, \cdot)$, it follows that

$$
\begin{aligned}
f(z+t(v-z)) & \leq f(z)+t\left[\int_{\Omega} \Psi(x, \nabla v) d x-\int_{\Omega} \Psi(x, \nabla z) d x\right]+r_{2} t \\
& \leq f(z)+\left(r_{1}+r_{2}\right) t
\end{aligned}
$$

whenever $z \in W_{0}^{1,1}(\Omega),\|z-u\|_{n /(n-1)} \leq \delta$ and $0 \leq t \leq \delta$. Given $\varepsilon>0$, we may also assume that $\delta<\varepsilon$. Then, if we set

$$
\mathcal{V}((z, \tau), t)=v-z,
$$

it follows that

$$
\begin{aligned}
& \|\mathcal{V}((z, \tau), t)-(v-u)\|_{\frac{n}{n-1}}<\varepsilon, \\
& f(z+t \mathcal{V}((z, \tau), t)) \leq f(z)+\left(r_{1}+r_{2}\right) t \leq \tau+\left(r_{1}+r_{2}\right) t,
\end{aligned}
$$

whenever $(z, \tau) \in \mathrm{B}_{\delta}((u, f(u))) \cap \operatorname{epi}(f)$ and $0 \leq t \leq \delta$. According to Definition 6, we have that

$$
f_{\varepsilon}^{0}(u ; v-u) \leq\left(r_{1}+r_{2}\right)
$$

and (2) follows by the arbitrariness of $\varepsilon, r_{1}$ and $r_{2}$.

In particular, we have $f^{0}(u ; v-u)<+\infty$ for all $v \in C_{c}^{1}(\Omega)$, which is dense in $W_{0}^{1,1}(\Omega)$. Since $f^{0}(u ; \cdot)$ is convex and lower semicontinuous in $L^{n /(n-1)}(\Omega)$, we deduce that either $f^{0}(u ; v)>-\infty$ for all $v \in W_{0}^{1,1}(\Omega)$ or $f^{0}(u ; v)=-\infty$ for all $v \in W_{0}^{1,1}(\Omega)$. From Theorem 3, we infer assertion $(c)$, whence assertion $(a)$, in the case $\partial f(u)=\varnothing$.

To show assertion $(b)$ and complete the proof of assertion $(a)$, consider $\mu \in \partial f(u)$. Since

$$
f^{0}(u ;-u) \geq\langle\mu,-u\rangle>-\infty,
$$

from (2), we infer that $g(x, u) u \in L^{1}(\Omega)$, which implies that $g(x, u) \in L^{1}(\Omega)$, according to Remark 1.

Let $v \in W_{0}^{1,1}(\Omega)$ with $\Psi(x, \nabla v) \in L^{1}(\Omega)$ and $[g(x, u)(v-u)]^{-} \in L^{1}(\Omega)$, which is equivalent to $[g(x, u) v]^{-} \in L^{1}(\Omega)$.

Since $\Psi(x, 0)=0$, we have that

$$
\left|\Psi\left(x, \nabla T_{k}(v)\right)\right| \leq|\Psi(x, \nabla v)| \quad \text { for all } k>0 .
$$

Therefore, $\Psi\left(x, \nabla T_{k}(v)\right) \in L^{1}(\Omega)$ and we can choose $T_{k}(v)$ as the test function in (2), obtaining

$$
\begin{aligned}
\int_{\Omega} \Psi\left(x, \nabla T_{k}(v)\right) d x-\int_{\Omega} \Psi(x, \nabla u) d x-\int_{\Omega} g(x, u)\left(T_{k}(v)-u\right) d x & \\
& \geq f^{0}\left(u ; T_{k}(v)-u\right) .
\end{aligned}
$$

We also infer that

$$
\lim _{k \rightarrow+\infty} \int_{\Omega} \Psi\left(x, \nabla T_{k}(v)\right) d x=\int_{\Omega} \Psi(x, \nabla v) d x .
$$


On the other hand, we have

$$
\begin{aligned}
g(x, u)\left(T_{k}(v)-u\right) & \geq-\left[g(x, u) T_{k}(v)\right]^{-}-g(x, u) u \\
& \geq-[g(x, u) v]^{-}-g(x, u) u
\end{aligned}
$$

and $\left(T_{k}(v)\right)$ is convergent to $v$ in $L^{n /(n-1)}(\Omega)$. Combining the lower semicontinuity of $f^{0}(u ; \cdot)$ with Fatou's lemma, we infer that

$$
\int_{\Omega} \Psi(x, \nabla v) d x-\int_{\Omega} \Psi(x, \nabla u) d x-\int_{\Omega} g(x, u)(v-u) d x \geq f^{0}(u ; v-u)
$$

and the proof of assertion $(a)$ is complete.

Taking into account Definition 7, we deduce that

$$
\int_{\Omega} \Psi(x, \nabla v) d x-\int_{\Omega} \Psi(x, \nabla u) d x-\int_{\Omega} g(x, u)(v-u) d x \geq\langle\mu, v-u\rangle
$$

and assertion $(b)$ also follows.

Proposition 4. Let $u \in W_{0}^{1,1}(\Omega)$ with $g(x, u) u \in L^{1}(\Omega)$ and let $\mu$ be in the dual space of $L^{n /(n-1)}(\Omega)$.

Then, $u$ is a minimum of the convex functional

$$
\left\{v \mapsto \int_{\Omega} \Psi(x, \nabla v) d x-\int_{\Omega} g(x, u) v d x-\langle\mu, v\rangle\right\}
$$

on the linear space $V_{u}$ if and only if $u$ is a minimum of the same functional on the convex set

$$
\left\{v \in W_{0}^{1,1}(\Omega): \Psi(x, \nabla v) \in L^{1}(\Omega),[g(x, u) v]^{-} \in L^{1}(\Omega)\right\} .
$$

Proof. Similar to before, we also have $g(x, u) \in L^{1}(\Omega)$. Assume now that $u$ is a minimum of the convex functional

$$
\left\{v \mapsto \int_{\Omega} \Psi(x, \nabla v) d x-\int_{\Omega} g(x, u) v d x-\langle\mu, v\rangle\right\}
$$

on the linear space $V_{u}$ and let $v \in W_{0}^{1,1}(\Omega)$ with $\Psi(x, \nabla v) \in L^{1}(\Omega)$ and $[g(x, u) v]^{-} \in$ $L^{1}(\Omega)$.

Since $T_{k}(v) \in V_{u}$, we have

$$
\begin{aligned}
\int_{\Omega} \Psi\left(x, \nabla T_{k}(v)\right) d x-\int_{\Omega} g(x, u) T_{k}(v) d x & -\left\langle\mu, T_{k}(v)\right\rangle \\
& \geq \int_{\Omega} \Psi(x, \nabla v) d x-\int_{\Omega} g(x, u) v d x-\langle\mu, v\rangle .
\end{aligned}
$$

Similar to before, we also have that

$$
\begin{aligned}
& \lim _{k \rightarrow+\infty} \int_{\Omega} \Psi\left(x, \nabla T_{k}(v)\right) d x=\int_{\Omega} \Psi(x, \nabla v) d x, \\
& \liminf _{k \rightarrow+\infty} \int_{\Omega} g(x, u) T_{k}(v) d x \geq \int_{\Omega} g(x, u) v d x,
\end{aligned}
$$

whence

$$
\begin{aligned}
\int_{\Omega} \Psi(x, \nabla v) d x-\int_{\Omega} g(x, u) v d x-\langle\mu, v\rangle & \\
& \geq \int_{\Omega} \Psi(x, \nabla v) d x-\int_{\Omega} g(x, u) v d x-\langle\mu, v\rangle .
\end{aligned}
$$


The converse is easily seen.

Proposition 5. Let $u \in W_{0}^{1,1}(\Omega)$ and let $\mu$ be in the dual space of $L^{n /(n-1)}(\Omega)$. Assume that $g(x, u) u \in L^{1}(\Omega)$ and that $u$ is a minimum of the convex functional

$$
\left\{v \mapsto \int_{\Omega} \Psi(x, \nabla v) d x-\int_{\Omega} g(x, u) v d x-\langle\mu, v\rangle\right\}
$$

defined on $V_{u}$. Suppose also that, for a.e. $x \in \Omega$, the function $\Psi(x, \cdot)$ is of class $C^{1}$.

Then,

$$
\Psi(x, \nabla u) \in L^{1}(\Omega), \quad \nabla_{\xi} \Psi(x, \nabla u) \in L_{l o c}^{1}\left(\Omega ; \mathbb{R}^{n}\right), \quad \nabla_{\xi} \Psi(x, \nabla u) \cdot \nabla u \in L^{1}(\Omega),
$$

and we have

$$
\begin{aligned}
& \int_{\Omega} \nabla_{\xi} \Psi(x, \nabla u) \cdot \nabla v d x-\int_{\Omega} g(x, u) v d x=\langle\mu, v\rangle \quad \text { for all } v \in C_{c}^{1}(\Omega), \\
& \int_{\Omega} \nabla_{\xi} \Psi(x, \nabla u) \cdot \nabla u d x-\int_{\Omega} g(x, u) u d x \leq\langle\mu, u\rangle .
\end{aligned}
$$

Moreover, we also have

$$
\begin{aligned}
& \nabla_{\xi} \Psi(x, \nabla u) \cdot \nabla v \in L^{1}(\Omega), \\
& \int_{\Omega} \nabla_{\xi} \Psi(x, \nabla u) \cdot(\nabla v-\nabla u) d x-\int_{\Omega} g(x, u)(v-u) d x \geq\langle\mu, v-u\rangle, \\
& \quad \text { for all } v \in W_{0}^{1,1}(\Omega) \text { with } \Psi(x, \nabla v) \in L^{1}(\Omega) \text { and }[g(x, u) v]^{-} \in L^{1}(\Omega) .
\end{aligned}
$$

Proof. Since $0 \in V_{u}$, we have $\Psi(x, \nabla u) \in L^{1}(\Omega)$. Moreover, similar to before, we have $g(x, u) \in L^{1}(\Omega)$. Let now $v \in W_{0}^{1,1}(\Omega)$ with $\Psi(x, \nabla v) \in L^{1}(\Omega)$ and $[g(x, u) v]^{-} \in L^{1}(\Omega)$. From Proposition 4 , we infer that $g(x, u) v \in L^{1}(\Omega)$ and, for every $\left.\left.t \in\right] 0,1\right]$, we have

$$
\int_{\Omega} \frac{\Psi(x, \nabla u+t(\nabla v-\nabla u))-\Psi(x, \nabla u)}{t} d x-\int_{\Omega} g(x, u)(v-u) d x \geq\langle\mu, v-u\rangle .
$$

On the other hand, the convexity of $\Psi(x, \cdot)$ yields

$$
\frac{\Psi(x, \nabla u+t(\nabla v-\nabla u))-\Psi(x, \nabla u)}{t} \leq \Psi(x, \nabla v)-\Psi(x, \nabla u) .
$$

Going to the upper limit as $t \rightarrow 0^{+}$, we infer that

$$
\begin{aligned}
& \nabla_{\xi} \Psi(x, \nabla u) \cdot(\nabla v-\nabla u) \in L^{1}(\Omega), \\
& \int_{\Omega} \nabla_{\xi} \Psi(x, \nabla u) \cdot(\nabla v-\nabla u) d x-\int_{\Omega} g(x, u)(v-u) d x \geq\langle\mu, v-u\rangle .
\end{aligned}
$$

In particular, the choice $v=0$ yields

$$
\begin{aligned}
& \nabla_{\xi} \Psi(x, \nabla u) \cdot \nabla u \in L^{1}(\Omega), \\
& \int_{\Omega} \nabla_{\xi} \Psi(x, \nabla u) \cdot \nabla u d x-\int_{\Omega} g(x, u) u d x \leq\langle u, u\rangle,
\end{aligned}
$$

whence $\nabla_{\xi} \Psi(x, \nabla u) \cdot \nabla v \in L^{1}(\Omega)$ for all $v \in W_{0}^{1,1}(\Omega)$ with $\Psi(x, \nabla v) \in L^{1}(\Omega)$ and $[g(x, u) v]^{-} \in L^{1}(\Omega)$.

We can also choose as $v$ any element of $C_{\mathcal{c}}^{1}(\Omega)$, which is a linear space. It follows that $\nabla_{\xi} \Psi(x, \nabla u) \in L_{l o c}^{1}\left(\Omega ; \mathbb{R}^{n}\right)$ and

$$
\int_{\Omega} \nabla_{\xi} \Psi(x, \nabla u) \cdot \nabla v d x-\int_{\Omega} g(x, u) v d x=\langle\mu, v\rangle
$$


for all $v \in C_{c}^{1}(\Omega)$.

For the last result of this section, we assume, more specifically with respect to $\left(G_{5}\right)$, that:

$\left(G_{6}\right) \quad$ If $n \geq 2$, there exist $\hat{\alpha} \in L^{1}(\Omega)$ and $\hat{\beta} \in \mathbb{R}$ such that

$$
|G(x, s)| \leq \hat{\alpha}(x)+\hat{\beta}|s|^{\frac{n}{n-1}} \quad \text { for a.a. } x \in \Omega \text { and all } s \in \mathbb{R} .
$$

Theorem 6. For every $(u, \tau) \in \operatorname{epi}(f)$ with $f(u)<\tau$, we have $\left|d \mathcal{G}_{f}\right|(u, \tau)=1$. In particular, for every $c \in \mathbb{R}$, the function $f$ satisfies condition $(\text { epi })_{c}$.

Proof. Consider, first, the case in which $g(x, u) u \in L^{1}(\Omega)$. Since

$$
\left|g(x, u)\left(u-T_{k}(u)\right)\right| \leq|g(x, u) u|,
$$

we have

$$
\lim _{k \rightarrow+\infty} \int_{\Omega} g(x, u)\left(u-T_{k}(u)\right) d x=0
$$

and, on the other hand, similar to before,

$$
\lim _{k \rightarrow+\infty} \int_{\Omega} \Psi\left(x, \nabla T_{k}(u)\right) d x=\int_{\Omega} \Psi(x, \nabla u) d x .
$$

Given $\varepsilon>0$ with $4 \varepsilon<\tau-f(u)$, let $k>0$ be such that

$$
\begin{aligned}
& \int_{\Omega} g(x, u)\left(u-T_{k}(u)\right) d x<\varepsilon, \\
& \int_{\Omega} \Psi\left(x, \nabla T_{k}(u)\right) d x<\int_{\Omega} \Psi(x, \nabla u) d x+\varepsilon, \\
& \left\|T_{k}(u)-u\right\|_{\frac{n}{n-1}}<\varepsilon .
\end{aligned}
$$

If we set $v=T_{k}(u)$, from Lemma 2, we infer that there exists $\delta>0$, with $\delta \leq 1$ and $\delta \leq \varepsilon$, such that

$$
\int_{\Omega} G(x, z+t(v-z)) d x \geq \int_{\Omega} G(x, z) d x-\varepsilon t,
$$

whenever $z \in W_{0}^{1,1}(\Omega),\|z-u\|_{n /(n-1)} \leq \delta$ and $0 \leq t \leq \delta$. Taking into account assumption $\left(G_{6}\right)$, we may also assume that

$$
\begin{aligned}
& \int_{\Omega} G(x, z) d x \geq \int_{\Omega} G(x, u) d x-\varepsilon, \\
& \|z-v\|_{\frac{n}{n-1}} \leq \varepsilon
\end{aligned}
$$

whenever $z \in W_{0}^{1,1}(\Omega)$ and $\|z-u\|_{n /(n-1)} \leq \delta$. 
From the convexity of $\Psi(x, \cdot)$, it follows that

$$
\begin{gathered}
f(z+t(v-z)) \leq \int_{\Omega} \Psi(x, \nabla z) d x+t\left[\int_{\Omega} \Psi(x, \nabla v) d x-\int_{\Omega} \Psi(x, \nabla z) d x\right] \\
\quad-\int_{\Omega} G(x, z+t(v-z)) d x \\
=\int_{\Omega} \Psi(x, \nabla z) d x+t\left[\int_{\Omega} \Psi(x, \nabla u) d x-\int_{\Omega} \Psi(x, \nabla z) d x\right] \\
+t\left[\int_{\Omega} \Psi(x, \nabla v) d x-\int_{\Omega} \Psi(x, \nabla u) d x\right]-\int_{\Omega} G(x, z+t(v-z)) d x \\
=f(z)+t(f(u)-f(z)) \\
+t\left[\int_{\Omega} \Psi(x, \nabla v) d x-\int_{\Omega} \Psi(x, \nabla u) d x\right] \\
\quad-\int_{\Omega} G(x, z+t(v-z)) d x+\int_{\Omega} G(x, z) d x \\
+t\left[\int_{\Omega} G(x, u) d x-\int_{\Omega} G(x, z) d x\right]
\end{gathered}
$$

whence

$$
\begin{aligned}
& \|[z+t(v-z)]-z\|_{\frac{n}{n-1}} \leq \varepsilon t \\
& f(z+t(v-z)) \leq f(z)+t(f(u)-f(z))+3 \varepsilon t
\end{aligned}
$$

whenever $z \in W_{0}^{1,1}(\Omega),\|z-u\|_{n /(n-1)} \leq \delta$ and $0 \leq t \leq \delta$.

If now $(z, \eta) \in \mathrm{B}_{\delta}(u, \tau) \cap$ epi $(f)$ and $t \in[0, \delta]$, it follows that

$$
\begin{aligned}
f(z+t(v-z)) & \leq \eta+t(f(u)-\eta)+3 \varepsilon t \\
& \leq \eta+t(f(u)-\tau+\delta+3 \varepsilon) \\
& \leq \eta+t(f(u)-\tau+4 \varepsilon) .
\end{aligned}
$$

In particular, if we set

$$
\mathcal{H}((z, \eta), t)=(z+t(v-z), \eta-t(\tau-f(u)-4 \varepsilon))
$$

for all $(z, \eta) \in \mathrm{B}_{\delta}(u, \tau) \cap \operatorname{epi}(f)$ and $t \in[0, \delta]$, we have $\mathcal{H}((z, \eta), t) \in \operatorname{epi}(f)$ and

$$
\begin{gathered}
\|\mathcal{H}((z, \eta), t)-(z, \eta)\| \leq t\left(\varepsilon^{2}+(\tau-f(u)-4 \varepsilon)^{2}\right)^{1 / 2}, \\
\mathcal{G}_{f}(\mathcal{H}((z, \eta), t))=\eta-t(\tau-f(u)-4 \varepsilon)=\mathcal{G}_{f}(z, \eta)-t(\tau-f(u)-4 \varepsilon),
\end{gathered}
$$

whence, by Proposition 2,

$$
\left|d \mathcal{G}_{f}\right|(u, \tau) \geq \frac{\tau-f(u)-4 \varepsilon}{\left(\varepsilon^{2}+(\tau-f(u)-4 \varepsilon)^{2}\right)^{1 / 2}} .
$$

Going to the limit as $\varepsilon \rightarrow 0^{+}$, we infer that $\left|d \mathcal{G}_{f}\right|(u, \tau)=1$ and the assertion follows in the case $g(x, u) u \in L^{1}(\Omega)$.

Consider now the other case, namely,

$$
\int_{\Omega} g(x, u) u d x=-\infty
$$


Let $M>0$ be such that

$$
\begin{aligned}
& \|u\|_{\frac{n}{n-1}} \leq M, \\
& \int_{\Omega} G(x, u) d x \leq M, \\
& \int_{\Omega} \Psi(x, \nabla z) d x \geq-M \quad \text { for all } z \in W_{0}^{1,1}(\Omega) .
\end{aligned}
$$

Given $r>f(u)+3 M+2$, by Lemma 2, there exists $\delta>0$, with $\delta \leq 1$, such that

$$
\int_{\Omega} G(x, z-t z) d x \geq \int_{\Omega} G(x, z) d x+r t
$$

whenever $z \in W_{0}^{1,1}(\Omega),\|z-u\|_{n /(n-1)} \leq \delta$ and $0 \leq t \leq \delta$. If $f(z) \leq \tau+\delta$, we also have

$$
-M-\int_{\Omega} G(x, z) d x \leq f(z) \leq \tau+1,
$$

whence

$$
\int_{\Omega} G(x, z) d x \geq-M-\tau-1 .
$$

Then, arguing, similar to before, with $v=0$, we infer that

$$
f(z-t z) \leq f(z)+t(f(u)-f(z))+t M-t r+t(2 M+\tau+1)
$$

whenever $z \in W_{0}^{1,1}(\Omega),\|z-u\|_{n /(n-1)} \leq \delta, f(z) \leq \tau+\delta$ and $0 \leq t \leq \delta$.

If now $(z, \eta) \in \mathrm{B}_{\delta}(u, \tau) \cap$ epi $(f)$ and $t \in[0, \delta]$, it follows that

$$
\begin{aligned}
f(z-t z) & \leq \eta+t(f(u)-\eta)+t(3 M+\tau+1-r) \\
& \leq \eta+t(f(u)+3 M+2-r) .
\end{aligned}
$$

In particular, if we set

$$
\mathcal{H}((z, \eta), t)=(z-t z, \eta-t(r-f(u)-3 M-2))
$$

for all $(z, \eta) \in \mathrm{B}_{\delta}(u, \tau) \cap \operatorname{epi}(f)$ and $t \in[0, \delta]$, we have $\mathcal{H}((z, \eta), t) \in \operatorname{epi}(f)$ and

$$
\begin{gathered}
\|\mathcal{H}((z, \eta), t)-(z, \eta)\| \leq t\left((M+1)^{2}+(r-f(u)-3 M-2)^{2}\right)^{1 / 2}, \\
\mathcal{G}_{f}(\mathcal{H}((z, \eta), t))=\eta-t(r-f(u)-3 M-2)=\mathcal{G}_{f}(z, \eta)-t(r-f(u)-3 M-2),
\end{gathered}
$$

whence, by Proposition 2,

$$
\left|d \mathcal{G}_{f}\right|(u, \tau) \geq \frac{r-f(u)-3 M-2}{\left((M+1)^{2}+(r-f(u)-3 M-2)^{2}\right)^{1 / 2}} .
$$

Going to the limit as $r \rightarrow+\infty$, we infer that $\left|d \mathcal{G}_{f}\right|(u, \tau)=1$ and the assertion follows also in this case.

\section{Proof of the Main Results}

In this section, we prove the results stated in the Introduction.

Proof of Theorem 1. Let $V$ be an $m$-dimensional linear subspace of $C_{c}^{1}(\Omega)$ and take a basis $\left\{e_{1}, \ldots, e_{m}\right\}$ in $V$. For every $\varrho>0$, the map

$$
\psi: S^{m-1} \rightarrow L^{\frac{n}{n-1}}(\Omega) \backslash\{0\}
$$


defined by

$$
\psi(\zeta)=\varrho \sum_{j=1}^{m} \zeta_{j} e_{j}
$$

is continuous and odd, while the functional

$$
\left\{v \mapsto \int_{\Omega} G(x, v) d x\right\}
$$

is continuous on $V$ by assumption $\left(\Psi G_{2}\right)$. If $\varrho>0$ is such that $\|\psi(\zeta)\|_{\infty}<r$ for all $\zeta \in S^{m-1}$, we also have

$$
\int_{\Omega} G(x, v) d x>0 \quad \text { for all } v \in \psi\left(S^{m-1}\right),
$$

whence

$$
\inf \left\{\int_{\Omega} G(x, v) d x: v \in \psi\left(S^{m-1}\right)\right\}>0 .
$$

On the other hand, the functional

$$
\left\{v \mapsto \int_{\Omega} \Psi(x, v) d x\right\}
$$

is convex and finite on $V$, hence continuous, according to ([9] Corollary 2.3). Therefore, we have

$$
\sup \left\{\int_{\Omega} \Psi(x, \nabla v) d x: v \in \psi\left(S^{m-1}\right)\right\}<+\infty .
$$

Let $\Lambda_{m}>0$ be such that

$$
\sup \left\{\int_{\Omega} \Psi(x, \nabla v) d x: v \in \psi\left(S^{m-1}\right)\right\}<\Lambda_{m} \inf \left\{\int_{\Omega} G(x, v) d x: v \in \psi\left(S^{m-1}\right)\right\}
$$

and let $\lambda \geq \Lambda_{m}$.

Of course, also $\Psi$ and $\lambda G$ satisfy $\left(\Psi G_{1}\right),\left(\Psi G_{2}\right),\left(\Psi G_{3}\right)$ and $\left(G_{4}\right)$, which implies $\left(G_{6}\right)$, hence $\left(G_{5}\right)$. Therefore, the functional

$$
\left.\left.f_{\lambda}: L^{\frac{n}{n-1}}(\Omega) \rightarrow\right]-\infty,+\infty\right],
$$

is lower semicontinuous, even and satisfies

$$
\sup _{\psi\left(S^{m-1}\right)} f_{\lambda}<0=f_{\lambda}(0) .
$$

Let $M>1$ be such that

$$
\lambda\|\hat{\beta}\|_{n}\|u\|_{\frac{n}{n-1}} \leq(M-1)\|\nabla u\|_{1} \quad \text { for all } u \in W_{0}^{1,1}(\Omega),
$$

where $\hat{\beta}$ is given by assumption $\left(G_{4}\right)$, and let $\alpha_{M}^{(2)}, \hat{\alpha}$ be as in assumptions $\left(\Psi G_{3}\right)$ and $\left(G_{4}\right)$. Then, for every $u \in W_{0}^{1,1}(\Omega)$, we have that

$$
\begin{aligned}
f_{\lambda}(u) & \geq M\|\nabla u\|_{1}-\left\|\alpha_{M}^{(2)}\right\|_{1}-\lambda\|\hat{\alpha}\|_{1}-\lambda\|\hat{\beta}\|_{n}\|u\|_{\frac{n}{n-1}} \\
& \geq\|\nabla u\|_{1}-\left\|\alpha_{M}^{(2)}\right\|_{1}-\lambda\|\hat{\alpha}\|_{1} .
\end{aligned}
$$

It follows that $f_{\lambda}$ is bounded from below and that, for every $c \in \mathbb{R}$, the set

$$
\left\{u \in W_{0}^{1,1}(\Omega): f_{\lambda}(u) \leq c\right\}
$$


is bounded in $W_{0}^{1,1}(\Omega)$, hence in $L^{n /(n-1)}(\Omega)$. Combining this fact with Corollary 2, we infer that $f_{\lambda}$ satisfies $(P S)_{c}$ for all $c \in \mathbb{R}$. Moreover, from Theorem 6 , we deduce that $f_{\lambda}$ satisfies $(\text { epi })_{c}$ for all $c \in \mathbb{R}$.

From Theorem 2, we infer that $f_{\lambda}$ admits $m$ distinct pairs $\left(u_{1},-u_{1}\right), \ldots,\left(u_{m},-u_{m}\right)$ such that $f_{\lambda}\left(u_{j}\right)<0$ and $\left|d f_{\lambda}\right|\left(u_{j}\right)=0$ for all $j=1, \ldots, m$.

By Theorem 3, we have that $0 \in \partial f_{\lambda}\left(u_{j}\right)$ for all $j=1, \ldots, m$. From Theorem 5 and Proposition 4 , we deduce that each $u_{j}$ is an energy critical point of $f_{\lambda}$.

Proof of Proposition 1. It is a particular case of Proposition 5 with $g$ replaced by $\lambda g$.

Author Contributions: All the authors contributed equally to this work. All authors have read and agreed to the published version of the manuscript.

Funding: This research received no external funding.

Conflicts of Interest: The authors declare no conflict of interest.

\section{References}

1. Rabinowitz, P.H. Minimax Methods in Critical Point Theory with Applications to Differential Equations; CBMS Regional Conference Series in Mathematics 65, Published for the Conference Board of the Mathematical Sciences; American Mathematical Society: Washington, DC, USA, 1986.

2. Struwe, M. Variational Methods; Ergebnisse der Mathematik und ihrer Grenzgebiete 34; Springer: Berlin, Germany, 2008.

3. Degiovanni, M.; Marzocchi, M.; Rădulescu, V.D. Multiple solutions of hemivariational inequalities with area-type term. Calc. Var. Partial Differ. Equ. 2000, 10, 355-387. [CrossRef]

4. Degiovanni, M.; Zani, S. Multiple solutions of semilinear elliptic equations with one-sided growth conditions. Math. Comput. Model. 2000, 32, 1377-1393. [CrossRef]

5. Degiovanni, M.; Schuricht, F. Buckling of nonlinearly elastic rods in the presence of obstacles treated by nonsmooth critical point theory. Math. Ann. 1998, 311, 675-728. [CrossRef]

6. Cellina, A. Some problems in the calculus of variations. Ann. Math. Silesianae 2017, 31, 5-55. [CrossRef]

7. Degiovanni, M.; Marzocchi, M. On the Euler-Lagrange equation for functionals of the calculus of variations without upper growth condition. SIAM J. Control Optim. 2009, 48, 2857-2870. [CrossRef]

8. Clark, D.C. A variant of the Ljusternik-Schnirelman theory. Indiana Univ. J. Math. 1972, 22, 65-74. [CrossRef]

9. Ekeland, I.; Témam, R. Convex Analysis and Variational Problems; Classics in Applied Mathematics 28; SIAM: Philadelphia, PA, USA, 1999.

10. Baroni, P.; Colombo, M.; Mingione, G. Nonautonomous functionals, borderline cases and related function classes. St. Petersburg Math. J. 2016, 27, 347-379.

[CrossRef]

11. Cupini, G.; Marcellini, P.; Mascolo, E. Regularity of minimizers under limit growth conditions. Nonlinear Anal. 2017, 153, 294-310. [CrossRef]

12. Růžička, M. Electrorheological Fluids: Modeling and Mathematical Theory; Lecture Notes in Mathematics 1748; Springer: Berlin, Germany, 2000.

13. Buttazzo, G. Semicontinuity, Relaxation and Integral Representation in The Calculus of Variations; Pitman Research Notes in Mathematics Series 207; Longman Scientific \& Technical: Harlow, UK, 1989.

14. Campa, I.; Degiovanni, M. Subdifferential calculus and nonsmooth critical point theory. SIAM J. Optim. 2000, 10, 1020-1048. [CrossRef]

15. Corvellec, J.-N.; Degiovanni, M.; Marzocchi, M. Deformation properties for continuous functionals and critical point theory. Topol. Methods Nonlinear Anal. 1993, 1, 151-171. [CrossRef]

16. Degiovanni, M.; Marzocchi, M. A critical point theory for nonsmooth functionals. Ann. Mat. Pura Appl. 1994, 167, 73-100. [CrossRef]

17. Ioffe, A.; Schwartzman, E. Metric critical point theory. I. Morse regularity and homotopic stability of a minimum. J. Math. Pures Appl. 1996, 75, 125-153.

18. Katriel, G. Mountain pass theorems and global homeomorphism theorems. Ann. L'Institut Henri Poincare (C) Non Linear Anal. 1994, 11, 189-209. [CrossRef]

19. De Giorgi, E.; Marino, A.; Tosques, M. Problemi di evoluzione in spazi metrici e curve di massima pendenza. Atti Della Accad. Dei Lincei Cl. Sci. Fis. Mat. Nat. Rend. 1980, 68, 180-187. 
20. Clarke, F.H. Optimization and Nonsmooth Analysis; Canadian Mathematical Society Series of Monographs and Advanced Texts; John Wiley \& Sons Inc.: New York, NY, USA, 1983.

21. Diestel, J. A survey of results related to the Dunford-Pettis property. In Proceedings of the Conference on Integration, Topology, and Geometry in Linear Spaces, Chapel Hill, NC, USA; Graves, W.H., Ed.; Contemporary Math. 2; American Mathematical Society: Washington, DC, USA, 1980; pp. 15-60. 